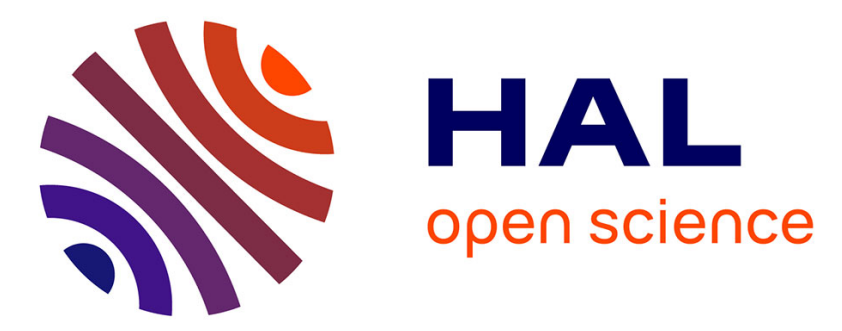

\title{
Memory Analysis and Optimized Allocation of Dataflow Applications on Shared-Memory MPSoCs
}

\author{
Karol Desnos, Maxime Pelcat, Jean-François Nezan, Slaheddine Aridhi
}

\section{To cite this version:}

Karol Desnos, Maxime Pelcat, Jean-François Nezan, Slaheddine Aridhi. Memory Analysis and Optimized Allocation of Dataflow Applications on Shared-Memory MPSoCs: In-Depth Study of a Computer Vision Application. Journal of Signal Processing Systems, 2015, 80 (1), pp.19-37. 10.1007/s11265-014-0952-6. hal-01083576v2

\section{HAL Id: hal-01083576 https://hal.science/hal-01083576v2}

Submitted on 10 Dec 2014

HAL is a multi-disciplinary open access archive for the deposit and dissemination of scientific research documents, whether they are published or not. The documents may come from teaching and research institutions in France or abroad, or from public or private research centers.
L'archive ouverte pluridisciplinaire $\mathbf{H A L}$, est destinée au dépôt et à la diffusion de documents scientifiques de niveau recherche, publiés ou non, émanant des établissements d'enseignement et de recherche français ou étrangers, des laboratoires publics ou privés. 


\title{
Memory Analysis and Optimized Allocation of Dataflow Applications on Shared-Memory MPSoCs
}

\section{In-Depth Study of a Computer Vision Application}

\author{
Karol Desnos • Maxime Pelcat . \\ Jean-François Nezan • Slaheddine Aridhi
}

Received: date / Accepted: date

\begin{abstract}
The majority of applications, ranging from the low complexity to very multifaceted entities requiring dedicated hardware accelerators, are very well suited for Multiprocessor Systems-on-Chips (MPSoCs). It is critical to understand the general characteristics of a given embedded application: its behavior and its requirements in terms of MPSoC resources.

This paper presents a complete method to study the important aspect of memory characteristic of an application. This method spans the theoretical, architectureindependent memory characterization to the quasi optimal static memory allocation of an application on a real shared-memory MPSoC. The application is modeled as an Synchronous Dataflow (SDF) graph which is used to derive a Memory Exclusion Graph (MEG) essential for the analysis and allocation techniques. Practical considerations, such as cache coherence and memory broadcasting, are extensively treated.

Memory footprint optimization is demonstrated using the example of a stereo matching algorithm from the computer vision domain. Experimental results show a reduction of the memory footprint by up to $43 \%$ compared to a state-of-the-art minimization technique, a throughput improvement of $33 \%$ over dynamic allocation, and the introduction of a tradeoff between multicore scheduling flexibility and memory footprint.
\end{abstract}

Keywords memory allocation · multiprocessor system-on-chip $\cdot$ stereo vision $\cdot$ synchronous dataflow

K. Desnos, M. Pelcat, J.-F. Nezan

IETR, INSA Rennes, UMR CNRS 6164, UEB

20 Avenue des Buttes de Coësmes, Rennes

E-mail: kdesnos, mpelcat, jnezan@insa-rennes.fr

S. Aridhi

Texas Instrument France

Avenue Jack Kilby, Villeneuve Loubet

E-mail: saridhi@ti.com

\section{Introduction}

Over the last decade, the popularity of data-intensive computer vision applications has rapidly grown. Research in computer vision traditionally aims at accelerating execution of vision algorithms with Desktop Graphics Processing Units (GPUs) or hardware implementations. The recent advances in computing power of embedded processors have made embedded systems promising targets for computer vision applications. Nowadays, computer vision is used in a wide variety of applications, ranging from driver assistance [1], to industrial control systems [20], and handheld augmented reality [34]. When developing data-intensive computer vision applications for embedded systems, addressing the memory challenges is an essential task as it can dramatically impact the performance of a system.

Indeed, the identification of the "memory wall" problem in 1995 [35] revealed memory issues as a major concern for developers of embedded systems. Memory issues strongly impact the quality and performance of an embedded system, as the area occupied by the memory can be as large as $80 \%$ of a chip and may be responsible for a major part of its power consumption [35, 14]. Despite the large silicon area allocated to memory banks, the amount of internal memory available on most embedded Multiprocessor Systems-on-Chips (MPSoCs) is still limited. Consequently, supporting the development of computer vision applications on highresolution images remains a challenging objective.

Prior work on memory issues for MPSoCs mostly focused on optimization techniques that minimize the amount of memory allocated to run an application, thus reducing the required memory real estate of the developed system $[21,12]$. These techniques may only be applied during late stages of the system design process 
because they rely on a precise knowledge of the system behavior, particularly the mapping and scheduling of the application tasks on the system processors.

This paper presents a complete method to study the memory characteristics of an application. This method spans from the theoretical and architecture independent memory characterization of an application to the quasi-optimal static memory allocation of this application on a real shared memory MPSoC. The objective of this paper is to show, through the example of a computer vision application, how this method can be used to efficiently address the memory challenges encountered during the development of an application on an embedded multicore processor.

The method proposed in this paper focuses on the characterization of applications described by a Dataflow Process Network (DPN). Representing an application with a DPN [19] consists of dividing this application into a set of processing entities, named actors, interconnected by a set of First In, First Out data queues (FIFOs). FIFOs allow the transmission of data tokens between actors. An actor starts its preemptionfree execution (it fires) when its incoming FIFOs contain enough data tokens. The number of data tokens consumed and produced during the execution of an actor is specified by a set of firing rules. The possibility of analyzing the DPNs as a result of their natural expressivity of parallelism make them particularly popular both for research [19,25] and commercial ends [17]. Indeed, it is this that makes DPN an attractive Model of Computation (MoC) to fully exploit the computing power offered by MPSoCs [25], GPUs, and manycore architectures [17].

The computer vision application which serves as our memory case study, as well as the MoC used to model it and the target MPSoC architecture are described in Section 2. The challenges targeted in this paper and the related works are presented in Section 3. Section 4 introduces a technique to bound the memory footprint of an application independent of device architecture. Then,

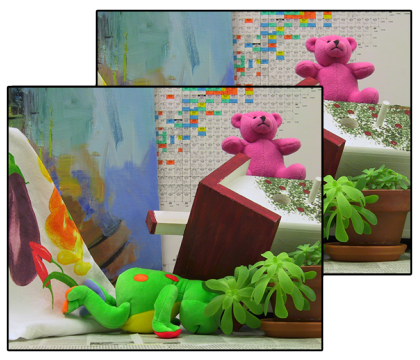

(a) Input Stereo Pair

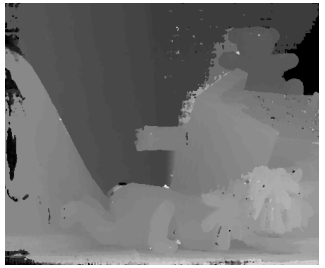

(b) Output Depth Map
Fig. 1 Stereo Matching Example
Section 5 presents several allocation strategies that offer a trade-off between application memory footprint and flexibility of the application multicore execution. In Section 6, we present our solutions to practical memory issues encountered when implementing a DPN on an MPSoC. Finally, experimental results of our method on the computer vision application are presented in Section 7 .

\section{Context}

This section introduces the context of our paper with a presentation of the semantics of the DPN MoC used, a description of the stereo matching application graph, and a presentation of the targeted architectures.

\subsection{Stereo matching}

The computer vision application studied in this paper is a stereo matching algorithm. Stereo matching algorithms are used in many computer vision applications such as $[1,11]$. As illustrated in Figure 1, the purpose of stereo matching algorithms is to process a pair of images (Figure 1(a)) taken by two cameras separated by a small distance in order to produce a disparity map (Fig. 1 (b)) that corresponds to the $3^{\text {rd }}$ dimension (the depth) of the captured scene. The large memory requirements of stereo matching algorithms make them interesting case studies to validate our memory analysis and optimization techniques.

Stereo matching algorithms can be sorted in two classes: global and local algorithms [30]. Global algorithms, such as graph cuts [27], are minimization algorithms that produce a depth map while minimizing a cost function on one or multiple lines of the input stereo pair. Despite the good quality of the results obtained with global algorithms, their high complexity make them unsuitable for real-time or embedded applications. Local algorithms independently match each pixel of the first image with a pixel selected in a restricted area of the second image [37]. The selection of the best match for each pixel of the image is usually based on a correlation calculus.

The stereo matching algorithm studied in this paper is the algorithm proposed by Zhang et al. in [37]. The low complexity, the high degree of parallelism, and the good accuracy of the result make this algorithm an appropriate candidate for implementation on an embedded MPSoC. The dataflow model of this algorithm is detailed in Section 2.2 


\subsection{Synchronous Dataflow (SDF)}

Synchronous Dataflow (SDF) [18] is the most commonly used DPN Model of Computation (MoC). In an SDF graph, the production and consumption token rates set by the firing rules of the actors are fixed scalars. This property allows a static analysis of an SDF graph during the application compilation. Static analyses can be used to ensure consistency and schedulability properties that imply deadlock-free execution of the application and bounded FIFO memory needs. If an SDF graph is consistent and schedulable, a fixed sequence of actor firings can be repeated indefinitely to execute the graph. This minimal sequence is deduced from the token exchange rates of the graph and is called graph iteration [19].

An example of an SDF graph with 5 actors is given in Figure 2. FIFOs are labeled with their token production and consumption rates. A FIFO with a dot signifies that initial tokens are present in the FIFO queue when the system starts to execute. The number of initial tokens is specified by the $x N$ label. Initial tokens are a semantic element of the SDF MoC that makes communication possible between successive iterations of the graph execution; they are often used to pipeline the execution of applications described with SDF graphs [18]. Actors have no states in the SDF MoC, consequently if enough data tokens are available, an actor can start several executions in parallel. For example in Figure 2, actor $A$ produces enough data tokens for actor $B$ to be executed twice in parallel. Hence, the SDF MoC naturally expresses the parallelism of an application. However, because of its self-loop FIFO, the two firings of actor $C$ cannot be executed simultaneously since the second firing requires data tokens produced by the first. Assigning a static order to the firings of the actors on the cores of a target architecture is called scheduling the application.

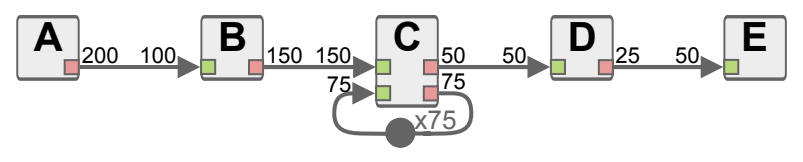

Fig. 2 SDF graph

Our SDF graph of the stereo matching algorithm is presented in Figure 3. For the sake of readability, all the token production and consumption rates displayed in the SDF graph are simplified and should be multiplied by the number of input image pixels to obtain the real exchange rates. Below each actor, in bold, is a repetition factor which indicates the number of executions of this actor during each iteration of the graph. This number of executions is deduced from the data productions and consumptions of actors. Two parameters are used in the graph: NbDisparities which represents the number of distinct values that can be found in the output disparity map, and NbOffsets which is a parameter influencing the size of the pixel area considered for the correlation calculus of the algorithm [37]. The SDF graph contains 12 distinct actors:

- ReadRGB produces the 3 color components of an input image by reading a stream or a file. This actor is called twice: once for each image of the stereo pair.

- BrdX is a broadcast actor. Its only purpose is to duplicate on its output ports the data token consumed on its input port.

- GetLeft gets the RGB left view of the stereo pair.

- RGB2Gray converts an RGB image into its grayscale equivalent.

- Census produces an 8-bit signature for each pixel of an input image. This signature is obtained by comparing each pixel to its 8 neighbors: if the value of the neighbor is greater than the value of the pixel, one signature bit is set to 1 ; otherwise, it is set to 0 .

- CostConstruction is executed once per possible disparity level. By combining the two images and their census signatures, it produces a value for each pixel that corresponds to the cost of matching this pixel from the first image with the corresponding pixel in the second image shifted by a disparity level.

- ComputeWeights produces 3 weights for each pixel, using characteristics of neighboring pixels. ComputeWeights is executed twice for each offset: once considering a vertical neighborhood of pixels, and once with a horizontal neighborhood.

- AggregateCosts computes the matching cost of each pixel for a given disparity. Computations are based on an iterative method that is executed NbOffsets times.

- DisparitySelect produces a disparity map by computing the disparity of the input cost map from the lowest matching cost for each pixel. The first input cost map is provided by an AggregateCosts actor and the second input cost map is the result of a previous comparison.

- RoundBuffer forwards the last disparity map consumed on its input port to its output port.

- MedianFilter applies a $3 \times 3$ pixels median filter to the input disparity map to smooth the results.

- Display displays the result of the algorithm or writes it in a file.

This SDF description of the algorithm provides a high degree of parallelism since it is possible to execute in 


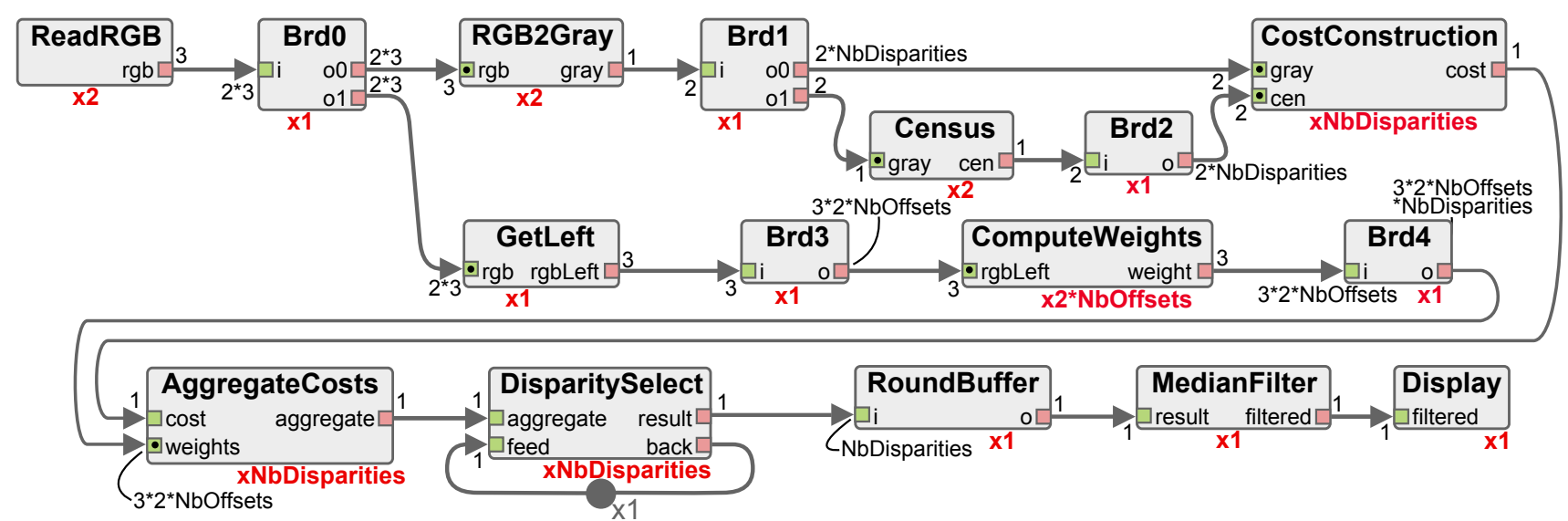

Fig. 3 Stereo-matching SDF graph. All rates are implicitly multiplied by the picture size.

parallel the repetitions of the three most computationally intensive actors, namely CostConstruction, AggregateCosts, and ComputeWeights. A detailed description of the original stereo matching algorithm can be found in [37] and our open-source SDF implementation is available online [8].

\subsection{Target architectures}

In this paper, we consider the implementation of the stereo matching algorithm on two multicore architectures:

The ${ }^{7}$-3610QM is a multicore Central Processing Unit (CPU) manufactured by Intel [15]. This 64bit processor contains 4 physical hyper-threaded cores that are seen as 8 virtual cores from the application side. This CPU has a clock speed of between $2.3 \mathrm{GHz}$ and 3.3GHz. Using virtual memory management technique, this CPU provides virtually unlimited memory resources to the applications it executes.

The TMS320C6678 is multicore Digital Signal Processor (DSP) manufactured by Texas Instruments [32]. This MPSoC contains 8 C66x DSPs, each running at $1.0 \mathrm{GHz}$ on our experimental evaluation module. Although the size of the addressable memory space is 8Gbytes, the evaluation module contains only 512 Mbytes of shared memory.

Contrary to the Intel's CPU, the TMS320C6678 does not have a hardware cache coherence mechanism to manage the private caches of each of its 8 cores. Consequently, it is the developer's responsibility to use writeback and invalidate functions to make sure that data stored in the two levels of private cache of each core is coherent.

The diverse memory characteristics and constraints of the two architectures must be taken into account when implementing an application. Section 3 presents the memory challenges encountered when implementing the stereo matching application on these two architectures.

\section{Challenges and Related Works}

This section presents the 3 main challenges addressed in the paper and their related work.

\subsection{Memory reuse}

To our knowledge, minimizing the memory footprint of dataflow applications is usually achieved by using FIFO dimensioning techniques $[24,29,22,2]$. FIFO dimensioning techniques consist of finding a schedule of the application that minimizes the memory space allocated to each FIFO of the SDF graph. For example, considering actors $B$ and $C$ from the graph of Figure 2, if the two repetitions of $B$ are scheduled before the two repetitions of $C(B B C C)$, then the FIFO between the two actors must be allocated enough memory to store 300 data tokens. However, if the 2 executions of $B$ and $C$ are interleaved $(B C B C)$ then only 150 data tokens need to be stored in the FIFO. This technique is used in the most popular dataflow frameworks such as SDF3 [29], Ptolemy II [24], or Kalray's dataflow tool chain [3].

The main drawback of FIFO dimensioning techniques is that they do not consider the reuse of memory since each FIFOs is allocated in a dedicated memory space. For example, if FIFO dimensioning is applied to the example of Figure 2, even though FIFOs $A B$ and $D E$ are never full simultaneously, they will not be allocated in overlapping memory spaces. Hence, FIFO dimensioning often results in wasted memory space [21]. 
As presented in Sections 4 to 6 , memory reuse is a key aspect of the memory analysis and optimization techniques presented in this paper. Moreover, contrary to most memory optimization techniques for SDF graphs that consider only monocore architectures [21, $29,22]$, our method focuses on shared memory multicore processors.

\subsection{Broadcast memory waste}

An important challenge when implementing the stereo matching application is the explosion of the memory space requirements caused by the broadcast actors. For example in Figure 3, with NbOffsets $=5, \mathrm{NbDispari-}$ ties $=60$ and a resolution of $450^{*} 375$ pixels, the broadcast actor Brd4 produces $3 * 2 *$ NbOffsets $*$ NbDisparities $*$ resolution float values, or $1.13 \mathrm{Gbytes}$ of data. Beside the fact that this footprint alone largely exceeds the 512 Mbytes capacity of the multicore DSP, this amount of memory is a waste as it consists only of 60 duplications of the 19.3Mbytes of data produced by the firings of the ComputeWeights actor.

Non-destructive reads, or FIFO peeking, is a wellknown way to read data tokens without consuming them, hence avoiding the need for broadcast actors [13]. Unfortunately, this technique cannot be applied without considerably modifying the underlying SDF MoC. Indeed, in the stereo matching example, using FIFO peeking would mean that the AggregateCosts only performs peeks and never consumes data tokens on its weights input port. Consequently, tokens would accumulate indefinitely on the FIFO connected to this port.

In Section 6, we propose a non-invasive addition to the SDF MoC to solve the broadcast issue.

\subsection{Cache coherence}

Cache management is a key challenge when implementing an application on a multicore target without automatic cache coherence. Indeed, as shown in [33], the use of cache dramatically improves the performance of an application on multi-DSP architectures, with execution times up to 24 times shorter that without cache.

An automatic method to insert calls to writeback and invalidate functions in code generated from a SDF graph is presented in [33]. As depicted in Figure 4, this method is applicable for shared memory communications between two processing elements. Actors $A$ and $B$ both have access to the shared memory addresses where data tokens of the $A B$ FIFO are stored. The synchronization between cores is ensured by the Send and Recv actors which can be seen as post and pend semaphore

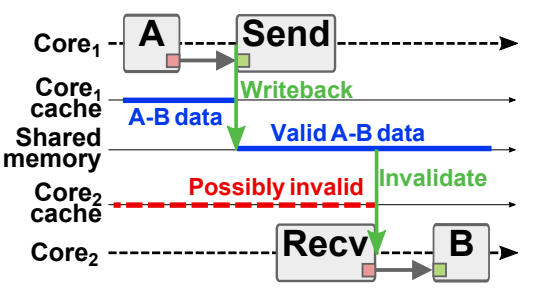

Fig. 4 Cache coherence solution without memory reuse

operations respectively. A writeback call is inserted before the Send operation to make sure that all $A B$ data tokens from Core $_{1}$ cache are written back in the shared memory. Similarly, an invalidate call is inserted after the Recv operation to make sure that cache lines corresponding to the address range of buffer $A B$ are removed from Core $_{2}$ cache.

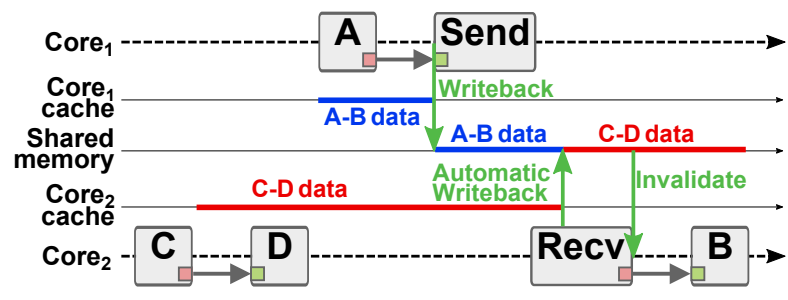

Fig. 5 Cache coherence issue with memory reuse

As depicted in Figure 5, a problem arises if the method presented in [33] is used jointly with memory reuse techniques. In this example, overlapping memory spaces are used to store data tokens of two FIFOs: $A B$ and $C D$. After the firings of actors $C$ and $D$, the cache memory of $\mathrm{Core}_{2}$ is "dirty", containing data tokens of the $C D$ FIFO that were not written back in the shared memory. Because these data tokens are "dirty", the local cache manager might generate an automatic writeback to put new data in the cache. If however, as in the example, this automatic writeback occurs after the writeback from Core $_{1}$, then the $C D$ data tokens will overwrite $A B$ tokens in the shared memory, thus corrupting the data accessed by actor $B$.

In addition to the memory reuse techniques presented in Sections 4 and 5, we propose a solution to generate code for cache-incoherent multicore architecture in Section 6.

\section{Memory Bounds}

Bounding the amount of memory needed to implement an application on a targeted multicore architecture is a key step of a development process. Indeed, memory upper and lower memory bounds are crucial pieces of 
information in the co-design process, as they allow the developer to adjust the size of the architecture memory according to the application requirements. Furthermore, as these bounds can be computed during the early development of an MPSoC, they might assist the developer in correct memory dimensioning (i.e. to avoid mapping an insufficient or an unnecessarily large memory chip).

The technique presented in this section is an analysis technique for deriving the memory allocation bounds (Figure 6) of an application modeled with an SDF graph.

\begin{tabular}{|c|c|c|}
$\begin{array}{c}\text { Insufficient } \\
\text { memory }\end{array}$ & $\begin{array}{c}\text { Possible } \\
\text { allocated memory }\end{array}$ & $\begin{array}{c}\text { Wasted } \\
\text { memory }\end{array}$ \\
\hline 0 & $\begin{array}{c}\text { Lower } \\
\text { Bound }\end{array}$ Allocational & $\begin{array}{c}\text { Upper } \\
\text { Bound }=\text { Allocation }\end{array}$ \\
\hline Worst Available
\end{tabular}

Fig. 6 Memory Bounds

This bounding technique is applicable in every stages of the development of an application, even when there is a complete abstraction of the system architecture. The bounding technique can be used both to predict memory requirements of an application in the early stages of its development, and to assess the quality of an allocation result during the implementation of an application. This bounding technique was first introduced in [6]. It is presented in this paper as a necessary first step to our memory reuse techniques as well as a way to assess the quality of our allocation results in Section 7.

\subsection{SDF graph pre-processing}

The first step to derive the memory bounds of an application consists of successively transforming its SDF graph into a single-rate SDF and into a Directed Acyclic Graph (DAG) so as to reveal its embedded parallelism and model its memory characteristics.

As exposed in [3], the transformation of an SDF graph into its equivalent single-rate SDF can be exponential in terms of number of actors. As a consequence, the method we propose should only be applied to SDF graphs with a relatively coarse grained description: graphs whose single-rate equivalent have at most hundreds of actors and thousands of singlerate buffers [6]. Despite this limitation, the single-rate transformation has proven to be efficient for many real applications, notably in the telecommunication [25] and the multimedia [7] domains.

\subsubsection{Pre-processing objectives}

In the context of memory analysis and allocation, the single-rate and the DAG transformations are applied with the following objectives:

- Expose data parallelism: Concurrent analysis of data parallelism and data precedence gives information on the lifetime of memory objects prior to any scheduling process. Indeed, two FIFOs belonging to parallel data-paths may contain data tokens simultaneously and are consequently forbidden from sharing a memory space. Conversely, two single-rate FIFOs linked with a precedence constraint can be allocated in the same memory space since they will never store data tokens simultaneously. In Figure 7 for example, FIFO $A B_{1}$ is a predecessor to $C_{1} D_{1}$. Consequently, these two FIFOs may share a common address range in memory.

- Break FIFOs into shared buffers: The memory needed to allocate each FIFO corresponds to the maximum number of tokens stored in the FIFO during an iteration of the graph [21]. However, in our method, the memory allocation can be independent from scheduling considerations. It is for this reason that FIFOs of undefined size before the scheduling step are replaced with buffers of fixed size during the transformation of the graph into a single-rate SDF.

- Derive an acyclic model: In the absence of a schedule, deriving a DAG permits the use of single-rate FIFOs that will be written and read only once per iteration of this DAG. Consequently, before a single rate FIFO is written and after it is read, its memory space will be reusable to store other objects.

\subsubsection{Graph transformations}

The first transformation applied to the input SDF graph to reveal parallelism is a conversion into a singlerate SDF graph. A single-rate SDF graph is an SDF graph where the production and consumption rates on each FIFO are equal. Each vertex of the single-rate SDF graph corresponds to a single actor firing from the SDF graph. This conversion is performed by computing the topology matrix [18], by duplicating actors by their number of firings, and by connecting FIFOs properly. For example, in Figure 7 , actors $B, C$, and $D$ are each split in two instances and new FIFOs are added to ensure the equivalence with the SDF graph of Figure 2. An algorithm to perform this conversion can be found in [28].

The second conversion consists of generating a Directed Acyclic Graph (DAG) by isolating one iteration of the algorithm. This conversion is achieved by ignoring FIFOs with initial tokens in the single-rate 


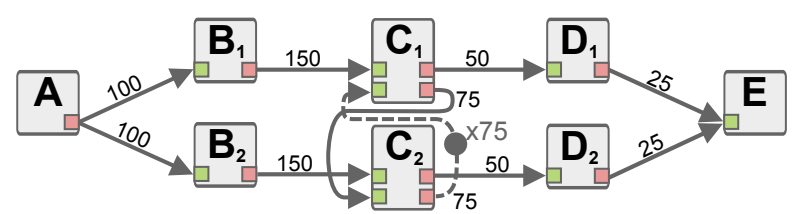

Fig. 7 Single-rate SDF graph. (Directed Acyclic Graph (DAG) if dotpoint FIFO is ignored)

SDF graph. In our example, this approach means that the feedback FIFO $C_{2} C_{1}$, which stores 75 initial tokens, is ignored. Our optimization technique does not allow the concurrent execution of successive iterations of the graph since the lifetime of each memory object is bounded by the span of a graph iteration. As presented in [18], delays can be added to acyclic data-paths of a dataflow graph in order to pipeline an application. By doing so, the developer can divide a graph into several unconnected graphs whose iterations can be executed in parallel, thus improving the application throughput. From the memory perspective, pipelining a graph will increase the graph parallelism and consequently the amount of memory required for its allocation. In the case of stereo matching, the addition of a pipeline stage after the AggregateCost actor leads to an increase of the memory footprint by $50 \%$. However, since the critical path is largely dominated by the most parallel actors, pipelining this application does not lead to a substantial throughput improvement.

\subsubsection{Memory objects}

The DAG resulting from the transformations of an SDF graph contains three types of memory objects:

- Communication buffers: The first type of memory object, which corresponds to the single-rate FIFOs of the DAG, are the buffers used to transfer data tokens between consecutive actors. In our approach, we consider that the memory allocated to these buffers is reserved from the execution start of the producer actor until the completion of the consumer actor. This choice is made to enable custom token accesses throughout actor firing time. As a consequence, the memory used to store an input buffer of an actor should not be reused to store an output buffer of the same actor. In Figure 7, the memory used to carry the 100 data tokens between actors $A$ and $B_{1}$ can not be reused, even partially, to transfer data from $B_{1}$ to $C_{1}$.

- Working memory of actors: The second type of memory object corresponds to the maximum amount of memory allocated by an actor during its execution. This working memory represents the memory needed to store the data used during the computations of the actor but does not include the input buffer nor the output buffer storage. In our method, we assume that an actor keeps exclusive access to its working memory during its execution. This memory is equivalent to a task stack space in an operating system.

- Feedback/Pipeline FIFOs: The last type of memory object corresponds to the memory needed to store feedback FIFOs ignored by the transformation of a single-rate SDF into a DAG. In Figure 7, there is a single feedback FIFO between $C_{2}$ and $C_{1}$. Each feedback FIFO is composed of 2 memory objects: the head and the (optional) body. The head of the feedback FIFO corresponds to the data tokens consumed during an iteration of the single-rate SDF. A head memory object may share memory space with any buffer that is both a successor to the actor consuming tokens from the feedback FIFO and a predecessor to the actor producing tokens on the feedback FIFO.

The body of the feedback FIFO corresponds to data tokens that remain in the feedback FIFO for several iterations of the graph before being consumed. A body memory object is needed only if the amount of delay on the feedback FIFO is greater than its consumption rate. A Body memory object must always be allocated in a dedicated memory space.

\subsection{Memory Exclusion Graph (MEG)}

Once an application SDF graph has been transformed into a DAG and all its memory objects have been identified, we derive a Memory Exclusion Graph (MEG) which will serve as a basis to our analysis and allocation techniques.

A Memory Exclusion Graph (MEG) is an undirected weighted graph denoted by $G=<V, E, w>$ where:

- $\mathbf{V}$ is the set of vertices. Each vertex represents an indivisible memory object.

- $\mathbf{E}$ is the set of edges representing the memory exclusions, i.e. the impossibility to share memory.

- w $: V \rightarrow \mathbb{N}$ is a function with $w(v)$ the weight of a vertex $v$. The weight of a vertex corresponds to the size of the associated memory object.

$-\mathbf{N}(\mathbf{v})$ the neighborhood of $v$, i.e. the set of vertices linked to $v$ by an exclusion $e \in E$. Vertices of this set are said to be adjacent to $v$.

- $|\mathbf{S}|$ the cardinality of a set $S .|V|$ and $|E|$ are the number of vertices and edges respectively of a graph.

$-\boldsymbol{\delta}(\mathbf{G})=\frac{2 \cdot|E|}{|V| \cdot(|V|-1)}$ the edge density of the graph corresponding to the ratio of existing exclusions to all possible exclusions.

Two memory objects of any type exclude each other (i.e. they can not be allocated in overlapping address 
ranges) if the DAG can be scheduled in such a way that both these memory objects store data simultaneously. Some exclusions are directly caused by the properties of the memory objects, such as exclusions between input and output buffers of an actor. Other exclusions result from the parallelism of an application, as is the case with the working memory of actors that might be executed concurrently because they belong to parallel data-paths.

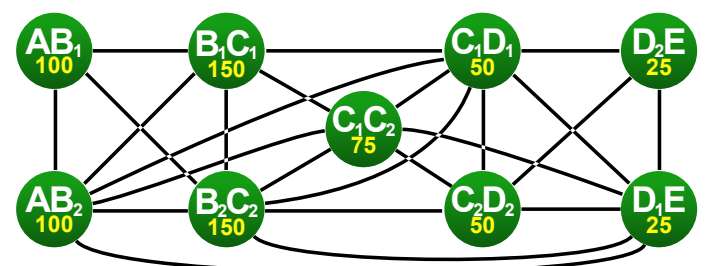

Fig. 8 Memory Exclusion Graph (MEG) derived from Fig. 2

The MEG presented in Figure 8 is derived from the SDF graph of Figure 2. The complete MEG contains 18 memory objects and 69 exclusions but, for clarity, only the vertices corresponding to the buffers between actors ( $1^{\text {st }}$ type memory objects) are presented. The values printed below the vertices names represent the weight $w$ of the memory objects.

The pseudo-code of an algorithm to build the complete MEG of an application is given in Figure 9.

The MEG obtained at this point of the method is a worst-case scenario since it models all possible exclusions for all possible schedules. As will be shown in Section 5, it is possible to update a MEG with scheduling information in order to reduce the number of exclusion, thus favoring memory reuse.

\subsection{Bounding techniques}

The upper and lower bounds of the static memory allocation of an application are a maximum and a minimum limit respectively to the amount of memory needed to run an application, as presented in Figure 6. The following four sections explain how the upper bound can be computed and give three techniques to compute the memory allocation lower bound. These three techniques offer a trade-off between accuracy of the result (Figure 10) and complexity of the computation.

\subsubsection{Least upper bound}

The least upper memory allocation bound of an application corresponds to the size of the memory needed
Input: a single-rate $\mathrm{SDF} \operatorname{sr} S D F=<A, F>$ with:

$A$ the set of actors

$F$ the set of FIFOs

Output: a Memory Exclusion Graph $M E G=<V, E, w>$

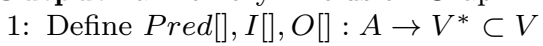

2: Sort $A$ in the DAG precedence order

3: for each $a \in A$ do

4: $\quad / *$ Process working memory of $a * /$

5: workingMem $\leftarrow$ new $v \in V$

6: $\quad w($ workingMem $) \leftarrow$ workingMemorySize $(a)$

7: $\quad$ for each $v \in V \backslash\{\operatorname{Pred}[a]$, workingMem $\}$ do

8: $\quad$ Add $e \in E$ between working Mem and $v$

9: end for

10: $\quad I[a] \leftarrow I[a] \cup\{$ workingMem $\}$

11: $\left.\quad\right|^{*}$ Process output buffers of $a * /$

12: for each $f \in(F \backslash$ feedbackFIFOs $) \cap$ outputs $(a)$ do

13: $\quad$ bufMem $\leftarrow$ new $v \in V$

14: $\quad w($ bufMem $) \leftarrow \operatorname{size}(f)$

15: $\quad$ for each $v \in V \backslash\{\operatorname{Pred}[a]$, buf $M e m\}$ do

16: $\quad$ Add $e \in E$ between buf Mem and $v$

17: $\quad$ end for

18: $\quad \operatorname{Pred}[$ consumer $(f)] \leftarrow \operatorname{Pred}[a] \cup I[a]$

19: $\quad I[$ consumer $(f)] \leftarrow I[$ consumer $(f)] \cup\{$ buf Mem $\}$

20: $\quad O[a] \leftarrow O[a] \cup\{b u f M e m\}$

21: end for

22: end for

23: /* Process Feedback FIFOs */

24: for each $f f \in F \cap$ feedbackFIFOs $(F)$ do

25: $\quad$ headMem $\leftarrow$ new $v \in V$

26: $\quad w($ headMem $) \leftarrow$ rate $(f f)$

27: $\quad$ set $\leftarrow(V \cap P[$ producer $(f f)]) \backslash P[$ consumer $(f f)]$

28: $\quad$ set $\leftarrow$ set $\backslash I[$ consumer $(f f)] \cup O[$ consumer $(f f)]$

29: for each $v \in V \backslash$ set do

30: $\quad$ Add $e \in E$ between headMem and $v$

31: end for

32: $\quad$ if rate $(f f)<$ delays $(f f)$ then

33: $\quad$ bodyMem $\leftarrow$ new $v \in V$

34: $\quad w($ bodyMem $) \leftarrow$ delays $(f f)-$ rate $(f f)$

35: $\quad$ for each $v$ in $V$ do

36: $\quad$ Add $e \in E$ between bodyMem and $v$

37: $\quad$ end for

38: end if

39: end for

Fig. 9 Building the Memory Exclusion Graph (MEG)

to allocate each memory object in a dedicated memory space. This allocation scheme is the least compact allocation possible as a memory space used to store an object would never be reused to store another.

Given a MEG $G$, its upper memory allocation bound is thus the sum of the weight of its vertices:

$$
\operatorname{Bound}_{\text {Max }}(G)=\sum_{v \in V} w(v)
$$

The upper bound for the MEG of Figure 8 is 725 units. As presented in Figure 10, using more memory than the upper bound means that part of the memory resources is wasted. Indeed, if a memory allocation uses an address range larger than this upper bound, some addresses within this range will never be read nor written. 


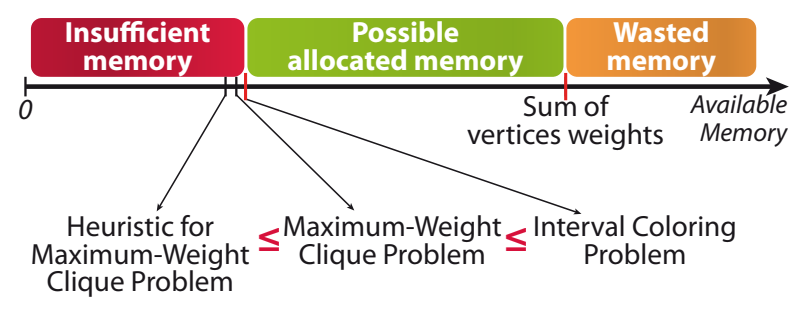

Fig. 10 Computing the lower memory bound

\subsubsection{Method 1 to compute the greatest lower bound - Interval Coloring Problem}

The greatest lower memory allocation bound of an application is the least amount of memory required to execute it. Finding this optimal allocation based on a MEG can be achieved by solving the equivalent Interval Coloring Problem [4, 12].

A k-coloring of a MEG is the association of each vertex $v_{i}$ of the graph with an interval $I_{i}=\{a, a+$ $1, \cdots, b-1\}$ of consecutive integers - called colors -, such that $b-a=w(v)$. Two distinct vertices $v_{i}$ and $v_{j}$ linked by an edge must be associated to non-overlapping intervals. Assigning an interval to a weighted vertex is equivalent to allocating a range of memory addresses to a memory object. Consequently, a k-coloring of a MEG corresponds to an allocation of its memory objects.

The Interval Coloring Problem consists of finding a $\mathrm{k}$-coloring of the exclusion graph with the fewest integers used in the $I_{i}$ intervals. This objective is equivalent to finding the allocation of memory objects that uses the least memory possible, thus giving the greatest lower bound of the memory allocation.

Unfortunately, as presented in [4], this problem is known to be NP-Hard, therefore it would be prohibitively long to solve for applications with hundreds or thousands of memory objects. Moreover, a sub-optimal solution to the Interval Coloring problem corresponds to an allocation that uses more memory than the minimum possible: more memory than the greatest lower bound. Consequently, a sub-optimal solution fails to achieve our objective which is to find a lower bound to the size of the memory allocated for a given application.

\subsubsection{Method 2 to compute a lower bound - Exact solution to the Maximum-Weight Clique Problem}

Since the greatest lower bound can not be found in reasonable time, we focus our attention on finding a lower bound close to the size of the optimal allocation. In [12], Fabri introduces another lower bound derived from an exclusion graph: the weight of the MaximumWeight Clique (MWC).

A clique is a subset of vertices that forms a subgraph within which each pair of vertices is linked with an edge. As memory objects of a clique can not share memory space, their allocation requires a memory as large as the sum of the weights of the clique elements, also called the clique weight. Subsets $S_{1}:=\left\{\mathrm{AB}_{1}, \mathrm{AB}_{2}\right.$, $\left.\mathrm{B}_{2} \mathrm{C}_{2}\right\}$ and $S_{2}:=\left\{\mathrm{C}_{1} \mathrm{D}_{1}, \mathrm{D}_{2} \mathrm{E}, \mathrm{C}_{2} \mathrm{D}_{2}, \mathrm{D}_{1} \mathrm{E}\right\}$ are examples of cliques in the MEG of Figure 8. Their respective weights are 350 and 150 . By definition, a single vertex can also be considered as a clique. A clique is called maximal if no vertex can be added to it to form a larger clique. In Figure 8, clique $S_{2}$ is maximal, but clique $S_{1}$ is not as $B_{1} C_{1}$ is linked to all the clique vertices and can therefore be added to the clique.

The Maximum-Weight Clique (MWC) of a graph is the clique whose weight is the largest of all cliques in the graph. Although this problem is also known to be NPHard, several algorithms have been proposed to solve it efficiently. In [23], Östergård proposes an exact algorithm which is, to our knowledge, the fastest algorithm for MEGs with an edge density under 0.80. For graphs with an edge density above 0.80 , a more efficient algorithm was proposed by Yamaguchi et al in [36]. Both algorithms are recursive and use a similar branch-andbound approach. Beginning with a subgraph composed of a single vertex, they search for the MWC $C_{i}$ in this subgraph. Then, a vertex is added to the considered subgraph, and the weight of $C_{i}$ is used to bound the search for a larger clique $C_{i+1}$ in the new subgraph. In [6], the two algorithms were implemented to compare their performances on exclusion graphs derived from different applications. In the exclusion graph of Figure 8, the MWC is $\left\{\mathrm{AB}_{2}, \mathrm{~B}_{1} \mathrm{C}_{1}, \mathrm{~B}_{2} \mathrm{C}_{2}, \mathrm{C}_{1} \mathrm{C}_{2}, \mathrm{C}_{1} \mathrm{D}_{1}\right\}$ with a weight of 525 units.

The weight of the MWC corresponds to the amount of memory needed to allocate the memory objects belonging to this subset of the graph. By extension, the allocation of the whole graph will never use less memory than the weight of its MWC. Therefore, this weight is a lower bound to the memory allocation and is less than or equal to the greatest lower bound, as shown in Figure 10.

\subsubsection{Method 3 to compute a lower bound - Heuristic for the Maximum-Weight Clique Problem}

Östergård's and Yamaguchi's algorithms are exact algorithms and not heuristics. Since the MWC problem is an NP-Hard problem, finding an exact solution in polynomial time can not be guaranteed. For this rea- 
son, we have developed a heuristic algorithm for the MWC problem.

The proposed heuristic approach, presented in Figure 11 , is an iterative algorithm whose basic principle is to remove a judiciously selected vertex at each iteration, until the remaining vertices form a clique.

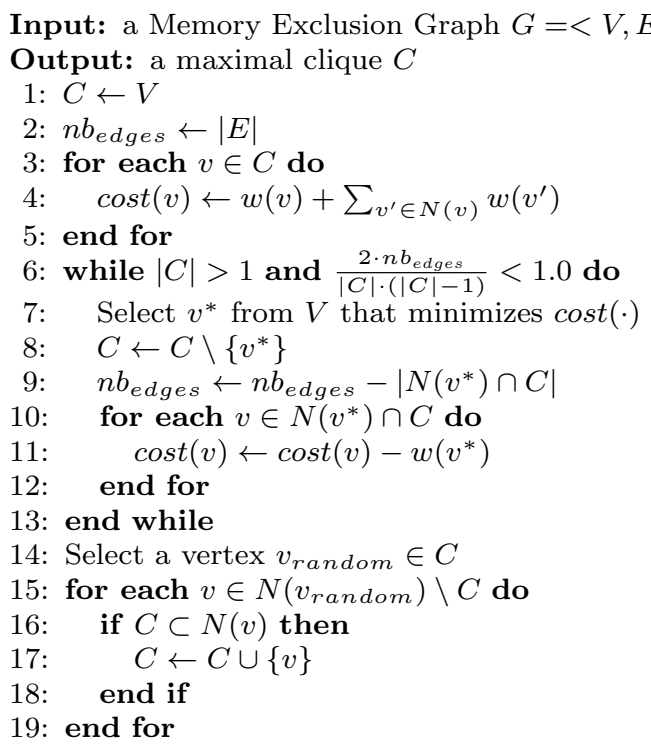

Fig. 11 Maximum-Weight Clique Heuristic Algorithm

Our algorithm can be divided into 3 parts:

- Initializations (lines 1-5): For each vertex of the MEG, the cost function is initialized with the weight of the vertex summed with the weights of its neighbors. In order to keep the input MEG unaltered through the algorithm execution, its set of vertices $V$ and its number of edges $|E|$ are copied in local variables $C$ and $n b_{\text {edges }}$.

- Algorithm core loop (lines 6-13): During each iteration of this loop, the vertex with the minimum cost $v^{*}$ is removed from $C$ (line 8). In the few cases where several vertices have the same cost, the lowest number of neighbor $|N(v)|$ is used to determine the vertex to remove. If the number of neighbors is equal, then selection is performed based on the smallest weight $w(v)$. By doing so, the number of edges removed from the graph is minimized and the edge density of the remaining vertices will be higher, which is desirable when looking for a clique. If there still are multiple vertices with equal properties, a random vertex is selected among them.

This loop is iterated until the vertices in subset $C$ become a clique. This condition is checked line 6 , by comparing 1.0 (the edge density of a clique) with the edge density of the subgraph of $G$ formed by the remaining vertices in $C$. To this purpose $n b_{\text {edge }}$, the number of edges of this subgraph, is decremented line 9 by the number of edges in $E$ linking the removed vertex $v^{*}$ to vertices in $C$. Lines 10 to 12 , the costs of the remaining vertices are updated for the next iteration.

- Clique maximization (lines 14-19): This last part of the algorithm ensures that the clique $C$ is maximal by adding neighbor vertices to it. To become a member of the clique, a vertex must be adjacent to all its members. Consequently, the candidates to join the clique are the neighbors of a vertex randomly selected in $C$. If a vertex among these candidates is linked to all vertices in $C$, it is added to the clique.

The complexity of this heuristic algorithm is of the order of $O\left(|N|^{2}\right)$, where $|N|$ is the number of vertices of the MEG.

In Table 1, the algorithm is applied to the MEG of Figure 8. For each iteration, the costs of the remaining vertices are given, and the vertex removed during the iteration is crossed out. The column $\delta(C)$ corresponds to the edge density of the subgraph formed by the remaining vertices. For example, in the first iteration, the memory object $D_{2} E$ has the lowest cost and is thus removed from the MEG. Before beginning the second iteration, the costs of memory objects $C_{1} D_{1}, C_{2} D_{2}$, and $D_{1} E$ are decremented by 25 : the weight of the removed memory object.

\begin{tabular}{|c|c|c|c|c|c|c|c|c|c|c|}
\hline \multirow{2}{*}{\multicolumn{2}{|c|}{$\operatorname{Iter} \delta(C$}} & \multicolumn{9}{|c|}{ Costs } \\
\hline & & $\mathrm{AB}_{1}$ & $\overline{\mathrm{AB}_{2}}$ & $\mathrm{~B}_{1} \mathrm{C}_{1}$ & $\mathrm{~B}_{2} \mathrm{C}_{2}$ & $\mathrm{C}_{1} \mathrm{C}_{2}$ & $\mathrm{C}_{1} \mathrm{D}_{1}$ & $\mathrm{C}_{2} \mathrm{D}_{2}$ & $\mathrm{D}_{2} \mathrm{E}$ & $\mathrm{D}_{1} \mathrm{E}$ \\
\hline 1 & 0.67 & 500 & 650 & 625 & 700 & 600 & 625 & 375 & 150 & 475 \\
\hline 2 & 0.75 & 500 & 650 & 625 & 700 & 600 & 600 & 350 & & 450 \\
\hline 3 & 0.81 & 500 & 650 & 625 & 650 & 550 & 550 & & & 400 \\
\hline 4 & 0.87 & 500 & 625 & 625 & 625 & 525 & 525 & & & \\
\hline 5 & 1.00 & & 525 & 525 & 525 & 525 & 525 & & & \\
\hline
\end{tabular}

Table 1 Algorithm proceeding for the MEG of Figure 8

In this simple example, the clique found by the heuristic algorithm and the exact algorithm are the same, and their weight also corresponds to the size of the optimal allocation. This example proves that, as shown in Figure 10, the result of the heuristic can be equal to the exact solution of the MWC problem, whose size can also equal that of the optimal allocation.

\section{Memory Allocation Strategies}

Given an initial MEG constructed from a non-scheduled DAG, we propose three possible implementation stages to perform the allocation of this MEG in shared memory: prior to any scheduling process, after an untimed 
multicore scheduling of actors, or after a timed multicore scheduling of the application. The scheduling flexibility resulting from the three alternatives are detailed in the following subsections.

\subsection{Memory Exclusion Graph (MEG) updates}

As presented in Section 4.2, the MEG built from the non-scheduled DAG is a worst-case scenario as it models all possible exclusions for all possible schedules of the application on any number of cores. If a multicore schedule of the application is known, this schedule can be used to update the MEG and lower its density of exclusions.

Scheduling a DAG on a multicore architecture introduces an order of execution of the graph actors, which is equivalent to adding new precedence relationships between actors. Adding new precedence edges to a DAG results in a decreased inherent parallelism of the application. For example, Figure 12 illustrates the new precedence edges that result from scheduling the DAG on 2 cores. In this example, Core $_{1}$ executes actors $B_{1}$, $C_{1}, D_{1}$ and $D_{2}$; and $C_{0 r e_{2}}$ executes actors $A, B_{2}, C_{2}$ and $E$.

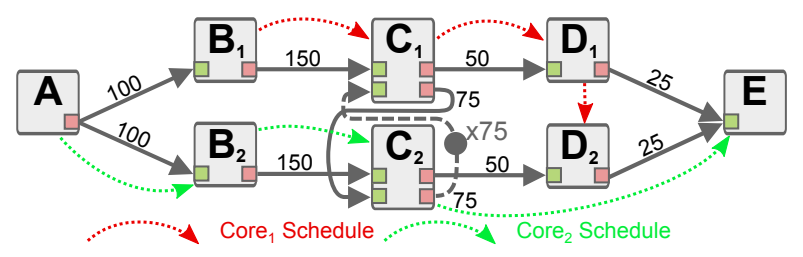

Fig. 12 Scheduled Single-rate SDF graph

As presented in Section 4.2, memory objects belonging to parallel data-paths may have overlapping lifetimes. Reducing the parallelism of an application results in creating new precedence paths between memory objects, thus preventing them from having overlapping lifetimes and removing exclusions between them. Since all the parallelism embedded in a DAG is explicit, the scheduling process cannot augment the parallelism of an application and cannot create new exclusions between memory objects. Figure 13 illustrates the updated MEG resulting from the multicore schedule of Figure 12.

A second update of the MEG is possible if a timed schedule of the application is available. A timed schedule is a schedule where not only the execution order of the actors is fixed, but also their absolute starting and ending times. Such a schedule can be derived if the exact, or the worst-case execution times of all actors

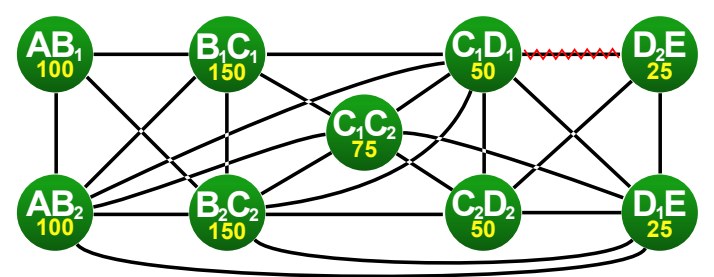

Fig. 13 MEG updated with schedule from Figure 12

are known at compile time [25]. Updating a DAG with a timed schedule consists of adding precedence edges between all actors with non-overlapping lifetimes.

Following assumptions made in Section 4.1, the lifetime of a memory object begins with the execution start of its producer, and ends with the execution end of its consumer. In the case of working memory, the lifetime of the memory object is equal to the lifetime of its associated actor. Using a timed schedule, it is thus possible to update a MEG so that exclusions remain only between memory objects whose lifetimes overlap. For example, the timed schedule of Figure 14(a) introduces a precedence relationship between actors $B_{2}$ and $C_{1}$ which translates into removing the exclusion between $\mathrm{AB}_{2}$ and $\mathrm{C}_{1} \mathrm{D}_{1}$ from the MEG.

\subsection{Static MEG allocation}

Allocating a MEG in memory consists of statically assigning an address range to each memory object. Possible approaches to perform the memory allocation are:

- Running an online allocation (greedy) algorithm. Online allocators assign memory objects one by one in the order in which they are fed to the allocator. Performance of online algorithms can be greatly improved by feeding the allocator with memory objects sorted in a smart order [7]. The most commonly used online allocators are the First-Fit (FF) and the Best-Fit (BF) algorithms [16]. FF algorithm consists of allocating an object to the first available space in memory of sufficient size. The BF algorithm works similarly but allocates each object to the available space in memory whose size is the closest to that of the allocated object.

- Running an offline allocation algorithm [14,21]. In contrast to online allocators, offline allocators have a global knowledge of all memory objects requiring allocation, thus making further optimizations possible.

- Coloring the MEG. Each vertex of the graph is associated with a set of colors such that two connected vertices have no color in common. The purpose of 
graph coloring technique is to minimize the total number of colors used in the graph [4].

- Using constraint programming [31] where memory constraints can be specified together with resource usage and execution time constraints.

In addition to these static allocation techniques, which are executed during the compilation of the application, dynamic allocation techniques can also be used. Dynamic allocation consists of allocating the memory objects of the MEG during the execution of the application. To keep the runtime overhead of dynamic allocation as low as possible, lightweight allocation algorithms such as the $\mathrm{FF}$ or the BF allocators are commonly used $[16,32]$.

\subsection{Pre-scheduling allocation}

Before scheduling the application, the MEG models all possible exclusions that may prevent memory objects from being allocated in the same memory space. Hence, a pre-scheduling MEG models all possible exclusions for all possible multicore schedules of an application. Consequently, a compile-time allocation based on a prescheduling MEG will never violate any exclusion for any valid multicore schedule of this graph on any sharedmemory architecture.

Since a compile-time memory allocation based on a pre-scheduling MEG is compatible with any multicore schedule, it is also compatible with any runtime schedule. The great flexibility of this first allocation approach is that it supports any runtime scheduling policy for the DAG and can accommodate any number of cores that can access a shared memory.

A typical scenario where this pre-scheduling compile-time allocation is useful is a multicore architecture implementation which runs multiple applications concurrently. In such a scenario, the number of cores used for an application may change at runtime to accommodate applications with high priority or those with high processing needs. The compile-time allocation relieves runtime management from the weight of a dynamic allocator while guaranteeing a fixed memory footprint for the application.

The downside of this first approach is that, as will be shown in the results of Section 7, this allocation technique requires substantially more memory than postscheduling allocators.

\subsection{Post-scheduling allocation}

Post-scheduling memory allocation offers a trade-off between amount of allocated memory and multicore sche- duling flexibility. The advantage of post-scheduling over pre-scheduling allocation is that updating the MEG greatly decreases its density which results in using less allocated memory [7].

Like pre-scheduling memory allocation, the flexibility of post-scheduling memory allocation comes from its compatibility with any schedule obtained by adding new precedence relationships to the scheduled DAG. Indeed, adding new precedence edges will make some exclusions useless but it will never create new exclusions. Any memory allocation based on the updated MEG of Figure 13 is compatible with a new schedule of the DAG that introduces new precedence edges. For example, we consider a single core schedule derived by combining schedules of Core $_{1}$ and Core $_{2}$ as follows $A$ $B_{2}, B_{1}, C_{1}, C_{2}, D_{1}, D_{2}$ and $E$. Updating the MEG with this schedule would result in removing the exclusions between $A B_{2}$ and $\left\{B_{1} C_{1}, C_{1} C_{2}, C_{1} D_{1}, D_{1} E\right\}$.

The scheduling flexibility for post-scheduling allocation is inferior to the flexibility offered by pre-scheduling allocation. Indeed, the number of cores allocated to an application may be only decreased at runtime for post-scheduling allocation while pre-scheduling allocation allows the number of cores to be both increased and decreased at runtime.

\subsection{Post-Timing allocation}

A MEG updated with a timed schedule has the lowest density of the three alternatives, which leads to the best results in terms of allocated memory size. However, its reduced parallelism makes it the least flexible scenario in terms of multicore scheduling and runtime execution.

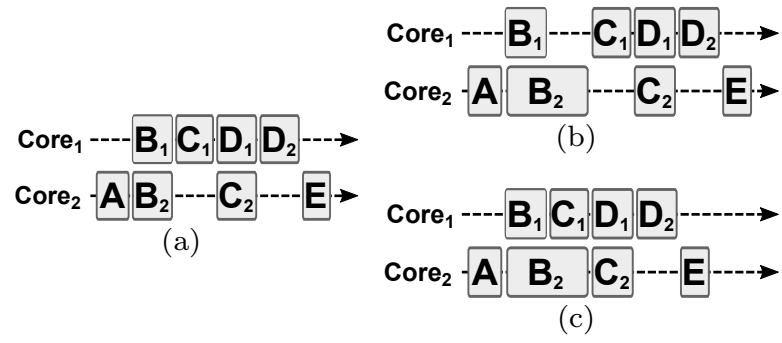

Fig. 14 Loss of runtime flexibility with timed allocation. (a) Timed schedule for the graph of Figure 12 .

(b)(c) Execution Gantt charts for timed and post-scheduling allocation with a doubled execution time for actor $\mathrm{B}_{2}$.

Figure 14 illustrates the possible loss of flexibility resulting from the usage of post-timing allocation. In the timed schedule of Figure 14(a), the same memory space can be used to store buffers $\mathrm{AB}_{2}$ and $\mathrm{C}_{1} \mathrm{D}_{1}$ since 
$\mathrm{B}_{2}$ execution ends before $\mathrm{C}_{1}$ execution starts. In Figures $14(\mathrm{c})$ and $14(\mathrm{~b})$, we consider that the execution time of actor $\mathrm{B}_{2}$ is double that of the timed schedule. With timed allocation (Figure 14(b)), the execution of $\mathrm{C}_{1}$ must be delayed until $\mathrm{B}_{2}$ completion, or otherwise $C_{1}$ might overwrite and corrupt data of the $A_{2}$ buffer. With post-scheduling allocation (Figure 14(c)), only the actor order on each core must be guaranteed. $\mathrm{C}_{1}$ can thus start its execution before $\mathrm{B}_{2}$ completion since buffers $A_{2}$ and $C_{1} D_{1}$ exclude each other in the corresponding MEG (Figure 13).

Although timed allocation provides the smallest memory footprints [7], its lack of runtime flexibility makes it a bad option for implementation. Nevertheless, computing the memory bounds for a MEG updated with a timed schedule is a convenient way to approximate the memory footprint that would be allocated by a dynamic allocator. Using dynamic allocation consists of dynamically allocating each buffer when it is first needed and freeing it when it has been consumed. Static timed allocation and dynamic allocation reach similar memory footprints as they both allow memory reuse as soon as the lifetime of a buffer is over.

A comparison of the three allocation strategies is available in [7] and their application to the stereo matching algorithm will be presented in Section 7 .

\section{Solutions to Implementation Issues}

\subsection{Zero-copy broadcasts}

The memory waste produced by the broadcast actors of an SDF graph is illustrated in Figure 15. As introduced in Section 3.2, the only purpose of the broadcast actor of Figure 15(a) is to duplicate the $n$ data tokens produced by actor $A$ to provide a copy of these data tokens to actors $B, C$, and $D$. In the corresponding MEG (Figure 15(b)), each FIFO connected to the broadcast actor is associated with a separate memory object of size $n$. Following the rules presented in Section 4.1, exclusions are added between all memory objects. Since the 4 memory objects form a clique, their allocation requires enough memory to store $4 * n$ data tokens. Since all 4 memory objects store identical data, this pattern can be seen to be a waste of memory.

During its execution, an SDF actor can use its input buffers as scratchpad memory and write new values in these memory spaces. Duplicating the broadcasted data tokens is thus necessary to make sure that the data in the input buffer of an actor is not corrupted by the activity of another actor.

Our solution to avoid the waste of memory caused by broadcast actors is to allow the developer to specify

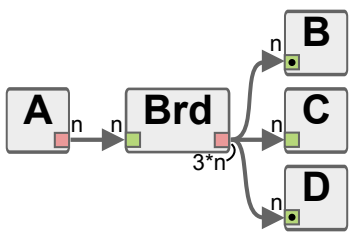

(a) SDF

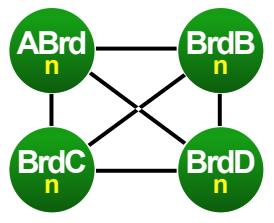

(b) MEG
Fig. 15 Broadcast memory waste

whether an actor uses its input buffers as scratchpad memories or if it only reads the consumed values. In Figure 15(a) and Figure 3, read only input ports are marked with a black dot within the port anchor. Because actors $B$ and $D$ have a read only input port, a private copy of the broadcasted data tokens is no longer needed and both actors can have a direct access to the $A B r d$ memory object. Actor $C$ however requires a private copy of the data tokens since it does not have a read only input port. Consequently, adding the read only information allows us to merge the memory objects $A B r d, B r d B$, and $B r d D$ as a single memory object of size $n$. As a result, only $2 * n$ memory units are needed to allocate the SDF graph of Figure 15(a), or half as much as the original memory requirement.

Contrary to FIFO peeking [13], our buffer merging technique does not require any change of the underlying SDF MoC. Similarly to annotations of imperative languages, marking an input port with the read only attribute does not have any effect on the behavior of the application. Indeed, read only attributes can be ignored during graph transformations and during the scheduling of the application and can be optionally used to reduce the memory footprint during the memory allocation process.

In addition to a drastic reduction of the memory footprint of the application, buffer merging also improves the performance of the application. Since the input buffers with a read only attribute are merged with the broadcasted buffer, the copy operation associated to these buffers is no longer needed. As shown in Section 7, these zero-copy broadcasts have a positive impact on the application performance both on the multicore DSP and the CPU targets.

\subsection{Automatic cache coherence}

The cache coherence mechanism presented in Section 3.3 is incompatible with memory reuse techniques. As presented in Figure 5, the insertion of writeback and invalidate calls around inter-core synchronization actors Send and Recv may result in data corruption in cases where a memory space is reused to store data to- 
kens from several buffers. This data corruption is caused by the automatic writeback of dirty lines of cache corresponding to a reused memory space.

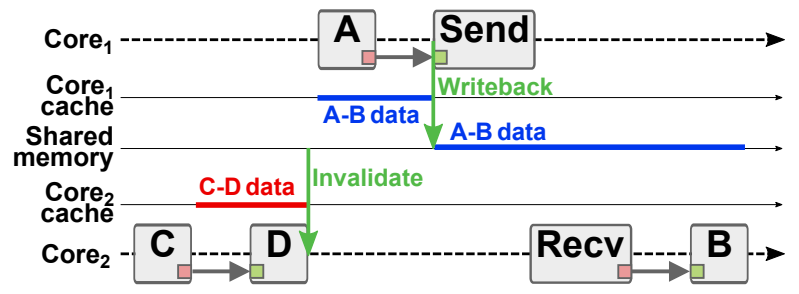

Fig. 16 Multicore cache coherence solution

Our solution to prevent unwanted writebacks is to make sure that no dirty lines of cache remain once the data tokens of a FIFO have been consumed. To this purpose, a call to the invalidate function is inserted for each buffer, after the firing of the actor consuming this buffer. As illustrated in Figure 16, the new calls to invalidate replace those inserted after the Recv synchronization actor.

As shown in Section 7, this solution allows us to activate the cache of the multicore DSP, leading to a huge improvement of the stereo-matching algorithm performance.

\section{Experiments}

\subsection{Hardware/software exploration workflow}

The stereo matching algorithm and the memory analysis and optimization presented in this paper were implemented within a rapid prototyping framework called Preesm. Preesm (the Parallel and Real-time Embedded Executives Scheduling Method) is an open source framework developed at the IETR for research and educational purposes [25]. Rapid prototyping consists of extracting information from a system in the early stages of its development. It enables hardware/software codesign and favors early decisions that improve system architecture efficiency.

Figure 17 illustrates the position of the memory analysis and optimization techniques in the rapid prototyping process of PREESM [25]. Inputs of the rapid prototyping process consist of: an algorithm model respecting the SDF MoC, an architecture model respecting the System-Level Architecture Model (S-LAM) semantics [26], and a scenario providing constraints and prototyping parameters. The scenario ensures the complete separation of algorithm and architecture models.

In PREESM, algorithm and architecture models first undergo transformations in preparation for the rapid

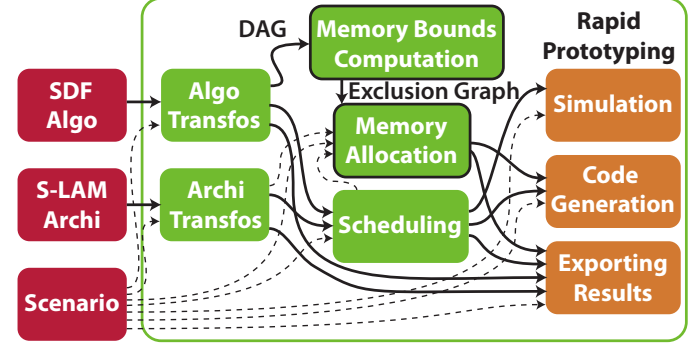

Fig. 17 Preesm rapid prototyping process

prototyping steps. Then, static multicore scheduling is executed to dispatch and schedule the algorithm actors to the architecture processing elements [25,5]. Finally, the multicore scheduling information is used to simulate the system behavior and to generate compilable code for the targeted architecture.

The complete independence between the architecture and algorithm models simplifies the porting of an application on different targets. For example, once the stereo-matching algorithm of Figure 3 was developed and tested on the Intel's CPU, it took only two hours to adapt the readRGB and display actors and generate a functional version for the 8 cores of the $C 6678$ multicore DSP. Afterwards, it takes only a few seconds to generate code for one of the two multicore architectures.

The PREESM project of the stereo matching application studied in this paper is available online [8].

7.2 Memory study of the stereo matching algorithm

Table 2 shows the memory characteristics resulting from the application of the techniques presented in this paper to the SDF graph of the stereo matching algorithm. The memory characteristics of the application are presented for 4 scenarios, each corresponding to a different implementation stage of the algorithm. The $|V|$ and $\delta(G)$ columns respectively give the number of memory objects and the density of exclusion of the MEG. The next two columns present the upper and lower allocation bounds for each scenario. Finally, the last two columns present the actual amount of memory allocated for each target architecture. The allocation results are expressed as the supplementary amount of memory allocated compared to the lower bound. These results were obtained with $\mathrm{NbOffsets}=5, \mathrm{Nb}$ Disparities $=60$ and a resolution of $450^{*} 375$ pixels.

\subsubsection{Effect of broadcast merging}

A comparison between the two pre-schedule scenarios of Table 2 reveals the impact of the merging of broad- 


\begin{tabular}{|l|c|c|r|r|r|r|}
\cline { 2 - 7 } \multicolumn{1}{c|}{} & \multicolumn{2}{c|}{ MEG } & \multicolumn{2}{c|}{ Bounds } & \multicolumn{2}{c|}{ Allocations ${ }^{2}$} \\
\hline \multicolumn{1}{c|}{ Scenarios } & $|V|$ & $\delta(G)$ & Upper & Lower & \multicolumn{1}{c|}{ i } & \multicolumn{1}{c|}{ C6678 } \\
\hline Pre-schedule & 1000 & 0.68 & $1524 \mathrm{MB}$ & $1378 \mathrm{MB}$ & $+0 \mathrm{kB}$ & $+52 \mathrm{kB}$ \\
Pre-schedule & 437 & 0.57 & $178 \mathrm{MB}$ & $104 \mathrm{MB}$ & $+168 \mathrm{kB}$ & $+695 \mathrm{kB}$ \\
Post-schedule & 437 & 0.47 & $178 \mathrm{MB}$ & $84 \mathrm{MB}$ & $+0 \mathrm{kB}$ & $+14 \mathrm{kB}$ \\
Post-timing & 437 & 0.39 & $178 \mathrm{MB}$ & $73 \mathrm{MB}$ & $+0 \mathrm{kB}$ & $+350 \mathrm{kB}$ \\
\hline 1: Merging of broadcasted buffers not applied in this scenario. \\
2: Relatively to the lower bound.
\end{tabular}

Table 2 MEGs characteristics and allocation results

casted buffers presented in Section 6.1. The first preschedule scenario presented in the table corresponds to the memory characteristics of the stereo matching application when buffer merging is not applied. With a memory footprint of 1378 Mbytes, this scenario forbid the allocation of the application in the 512Mbytes shared memory of the multicore DSP. The application of the buffer merging technique in the second scenario leads to a reduction of the memory footprint by $92 \%$, from 1378Mbytes to 104Mbytes.

Another positive aspect of the buffer merging technique is the simplification of the MEG. Indeed, 563 vertices are removed from the MEG as a result of the buffer merging technique. The computation of the memory bounds of the MEG and the allocation of the MEG in memory are both accelerated by a factor of 6 with the simplified MEG.

In addition to the large reduction of the memory footprint, buffer merging also has a positive impact on the application performance. On the $i 7$ multicore CPU, the stereo matching algorithm reaches a throughput of 1.84 frames per second (fps) when the broadcasted buffers are not merged, and a throughput of $3.50 \mathrm{fps}$ otherwise. Hence, the suppression of the memcpy results in a speedup ratio of $90 \%$. On the C6678 DSP, the suppression of the memcpy results in a speedup ratio of $40 \%$, rising from 0.24 fps to 0.34 fps.

\subsubsection{Memory footprints}

Results presented in Table 2 reveal the memory savings resulting from the application of the memory reuse techniques presented in this paper. 178Mbytes of memory are required for the allocation of the last three scenarios if, as in existing dataflow frameworks $[29,3$, 24], memory reuse techniques are not used. In the prescheduling scenario, memory reuse techniques lead to a reduction of the memory footprint by $41 \%$. This reduction of the memory footprint does not have any counterpart since the MEG is compatible with any schedule of the application (cf. Section 5). In the post-scheduling and in the post-timing scenarios, the memory footprints are respectively reduced by $53 \%$ and $59 \%$ compared to the memory footprint obtained without memory reuse.
The memory footprints allocated on the it CPU for these scenarios are optimal since the lower bounds for the MEGs and the allocation results are equal.

The memory footprints presented in Table 2 result from the allocation of the MEG with a Best-Fit (BF) allocator fed with memory objects sorted in the largestfirst order. A comparison of the efficiency of the different allocation algorithms that can be used to allocate a MEG is presented in [7].

Since all production and consumption rates of the stereo matching SDF graph are multiples of the image resolution, the memory footprints allocated with our method are proportional to the input image resolution. Using our memory reuse techniques, with $\mathrm{NbOffsets}=$ 5 and $\mathrm{Nb}$ Disparities $=60$, the $512 \mathrm{Mbytes}$ of the C6678 DSP allows the processing of stereo images with a resolution up to $720 \mathrm{p}$ (1280*720pixels). Without memory reuse, the maximum resolution that can fit within the multicore DSP memory is $576 \mathrm{p}$ ( $704^{*} 576$ pixels), which is 2.27 times less than when memory reuse is in effect.

\subsubsection{Cache activation}

Because of cache alignment constraints, the memory allocation results presented in Table 2 for the $C 6678$ multicore DSP are slightly superior to the results for the $i 7$ CPU. In order to avoid data corruption when the cache of the DSP is activated, the memory allocator must make sure that distinct buffers are never cached in the same line of cache. To this end, each buffer is allocated in a memory space aligned on the size of a L2 cache line: 128 bytes. On average, this policy results in an allocation increase of only $0.3 \%$ compared with the unaligned allocation of the $i 7 \mathrm{CPU}$.

As presented in Section 6.2, the insertion of writeback and invalidate calls in the code generated by PreEsm allows the activation of the caches of the C6678 multicore DSP. Without caches, the stereo-vision application reaches a throughput of $0.06 \mathrm{fps}$. When the caches of the DSP are activated, the application performance is increased by a factor of 5.7 and reaches $0.34 \mathrm{fps}$.

\subsection{Comparison with FIFO dimensioning techniques}

As presented in Section 3, FIFO dimensioning is currently the most widely used technique to minimize the memory footprint of applications modeled with a dataflow graph $[24,29,22]$. Table 3 compares allocation results of a FIFO dimensioning algorithm with those of our reuse technique for 4 application graphs. The FIFO dimensioning technique tested is presented in [29] and its implementation is part of the SDF3 framework [10]. 
The stereo graph is the application presented in Figure 3. The h263 enc. graph is a video encoder that was taken from the SDF3 database [10]. The sobel and chaotic graphs are a sobel video filtering application and a generator of chaotic sequences inspired by [9].

For a fair comparison, broadcast merging was simulated for the FIFO dimensioning technique by replacing broadcasted FIFOs with a parallel buffer of equivalent lifetime. Without this improvement, FIFO dimensioning techniques would produce similar results to those obtained with our reuse method before merging the broadcasted buffers.

\begin{tabular}{|c|c|c|c|c|}
\hline Graph & Upper Bound $^{1}$ & Pre-sched. ${ }^{1}$ & Post-sched. $^{1}$ & FIFO dim. \\
\hline stereo & $+109 \%$ & $+20 \%$ & $-15 \%$ & $0 \%$ \\
\hline h263 enc & $+116 \%$ & $-1 \%$ & $-17 \%$ & $0 \%$ \\
\hline sobel & $+46 \%$ & $-43 \%$ & $-43 \%$ & $0 \%$ \\
\hline chaotic & $+222 \%$ & $+77 \%$ & $+33 \%$ & $0 \%$ \\
\hline
\end{tabular}

1: Percentages are relative to the FIFO dimensioning result.

Table 3 Comparison of allocation results with FIFO dimensioning techniques from SDF3 [10].

Table 3 presents the results of the memory footprint size for the 4 scenarios. For each application, the results are expressed as percentages relative to the FIFO dimensioning case which is marked with $0 \%$. For the first 3 graphs, the post-scheduling scenario of our memory reuse technique offers the best results, with memory footprints up to $43 \%$ lower than the FIFO dimensioning technique. The FIFO dimensioning technique itself offers memory footprints on average $51 \%$ lower than the computed upper bound.

\subsubsection{Memory reuse technique limitations}

In Table 3, the FIFO dimensioning technique provides the best result for the chaotic graph, with $25 \%$ less memory than the post-scheduling memory allocation. This result reveals two current limitations of our memory reuse technique.

- Bad handling of divide/merge operations. During the single-rate transformation presented in Section 4.1, special actors are introduced to replace FIFOs with unequal production and consumption rates. These actors are responsible for dividing a buffer produced (or consumed) by an actor into subparts consumed (or produced) by other actors. Since the divided buffer and its subparts are input and output buffers of a single special actor, they exclude each other in the MEG and their allocation requires twice the size of the divided buffer in memory. This issue is not present in the FIFO dimensioning technique since buffer division is naturally implemented by successive data-token reads in FIFOs. The numerous divide and merge operation of the singlerate chaotic graph are thus responsible for its higher memory footprint.

- Absence of memory-aware scheduling process. As presented in Section 3, FIFO dimensioning techniques consists of finding the schedule of the application that minimizes the memory space allocated to each FIFO of the graph. In PREESM, the aim of the scheduling process is to minimize the latency of the schedule, independent of the memory allocation concerns. This policy often results in bad choices from the memory perspective, as is the case for the chaotic application where several actors producing large buffers are executed before any of the large buffers are consumed. With FIFO dimensioning techniques, the consuming actor of the large buffer would be scheduled immediately after its producer.

\subsection{Static vs dynamic memory allocation}

As presented in Section 5, similar footprints are obtained with dynamic allocation and static allocation in the post-timing scenario. In both cases, the memory allocated to a memory object can be reused as soon as the lifetime of this memory object ends. In this section we will show that although dynamic allocators provide low memory footprints, their runtime overhead and their unpredictability make them bad choices when compared to static allocation.

\subsubsection{Runtime overhead}

\begin{tabular}{|c|r|r|c|}
\cline { 2 - 3 } \multicolumn{1}{c|}{} & \multicolumn{2}{c|}{ Throughput } & \multicolumn{1}{c|}{} \\
\hline Target & Static Allocation & Dynamic Allocation & Overhead \\
\hline C6678 DSP & 0.39fps & 0.26fps & $32 \%$ \\
\hline
\end{tabular}

Table 4 Comparison of the stereo matching performance with static and dynamic allocations

Table 4 presents the performance of the stereo matching algorithm on the C6678 DSP. Two versions of the code were generated with PREESM: the first with post-scheduling allocation, and the second with dynamic memory allocation. For a fair comparison, the same schedule was used for both allocation strategies. To increase the application throughput in these tests, a software pipeline stage was added between the AggregateCost and the DisparitySelect actors.

Dynamic allocation had a negative impact on the performance of the application. On the C6678 DSP, the throughput reduction of $32 \%$ had three main sources: 
- The overhead of the dynamic allocator. Each time a memory object is dynamically allocated, the online allocation algorithm searches for an free space of sufficient size in the heap to store this memory object.

- The extra synchronization added to the generated code to dynamically support the merging of broadcasted buffers. A semaphore was associated with each broadcasted buffer and initialized with the number of actors accessing this buffer. Each actor accessing the broadcasted buffer decremented the value of the semaphore after its firing. When the semaphore value reached zero, a free operation was issued for the broadcasted buffer.

- The insertion of cache operations for the memory object pointers. Each time a buffer was allocated on one core, a writeback call was issued to ensure that the pointer value was written back in the shared memory. Similarly, a call to invalidate was required when a core accesses a buffer allocated on another core.

On the $i 7 \mathrm{CPU}$, the dynamic allocator overhead and the dynamic support for merged buffers also cause a throughput reduction of $22 \%$.

\subsubsection{Unpredictable footprint}

Although dynamic allocation provides memory footprints similar to post-timing allocation, the dynamic memory footprint cannot be bounded at compile time. To illustrate this issue, we measured the dynamic memory footprint of the stereo matching algorithm during 200 iterations of the graph execution. This experiment was conducted on the $C 6678$ by measuring, after each iteration, the maximum size of the heap on which the memory objects were dynamically allocated. The experiment was repeated 12 times with the same code but with different cache configurations (activation of level 1 and level 2 caches, location of the code, debug or release). These different configurations modify actor execution times and thus the order of memory allocation primitive calls. Each blue curve in Figure 18 represents the footprints measured during one of the 12 experiments.

This experiment shows that the dynamic memory footprint of an application increases during the first iterations. This increase of the memory footprint is caused by the fragmentation of the memory. Memory fragmentation happens when a free space in the heap is too small to allocate new memory objects. Because the DSP has no defragmenting process, the memory fragmentation tends to accumulate during the first it-

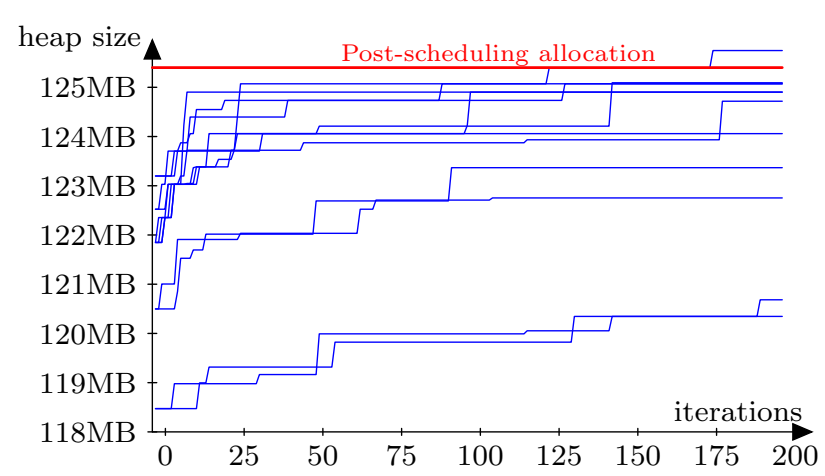

Fig. 18 Dynamic allocation: Heap size after $\mathrm{N}$ iterations. Each blue line represents the heap size for an execution of the stereo matching application.

erations of the application, which results in an increase of the heap size.

The memory footprints measured in Figure 18 range between 118.5Mbytes and 125.7Mbytes. The $6 \%$ difference between these two values illustrates the unpredictability of the dynamic memory footprint of applications. Finally, post-scheduling allocation for this schedule results in a memory footprint of $125.4 \mathrm{Mbytes}$. Consequently, these experiments show that despite a slight reduction in the memory footprint with dynamic allocation, the exact memory footprint cannot be predicted with dynamic allocation and this dynamic footprint might even exceed its static equivalent.

\section{Conclusion}

In this paper, we have proposed new techniques to analyze and optimize the memory characteristics of applications modeled with SDF graphs. These techniques address key memory challenges encountered throughout the development of a system: from the estimation of the application memory footprint in the early stages of development, to the reduction of this memory footprint during the application implementation on a shared memory MPSoC. These techniques are the first to exploit memory reuse for the allocation of dataflow graphs in a multicore context. Through the application of our techniques on a state-of-the-art computer vision application, we have demonstrated the efficiency of these techniques and how they may be used to implement a data-intensive application on real MPSoCs with limited memory resources. Our experimental results showed that static allocation and memory reuse techniques significantly reduce the memory footprints of applications. Specifically, the memory footprint was reduced by a factor 18 on the stereo matching algorithm and up to $43 \%$ less memory than state-of-the-art min- 
imization techniques. We also showed the positive impact of our optimizations on application performance, with a throughput improvement of $33 \%$ compared to dynamic allocation techniques.

Future work on this subject may include the extension of our analysis and optimization techniques to support targets with distributed memory such as manycore architectures. Another potential direction of interest is the design of an iterative scheduling process that uses memory bounds to allow a trade-off between application latency and memory footprint.

\section{References}

1. Arndt, O., Becker, D., Banz, C., Blume, H.: Parallel implementation of real-time semi-global matching on embedded multi-core architectures. In: Embedded Computer Systems: Architectures, Modeling, and Simulation (SAMOS XIII) (2013)

2. Benazouz, M., Marchetti, O., Munier-Kordon, A., Urard, P.: A new approach for minimizing buffer capacities with throughput constraint for embedded system design. In: Computer Systems and Applications (AICCSA), 2010 IEEE/ACS (2010)

3. Bodin, B., Munier-Kordon, A., de Dinechin, B.: Kperiodic schedules for evaluating the maximum throughput of a synchronous dataflow graph. In: Embedded Computer Systems (SAMOS) (2012)

4. Bouchard, M., Angalović, M., Hertz, A.: About equivalent interval colorings of weighted graphs. Discrete Appl. Math. (2009). DOI 10.1016/j.dam.2009.04.015

5. Boutellier, J.: Quasi-static scheduling for fine-grained embedded multiprocessing. Ph.D. thesis, University of Oulu (2009)

6. Desnos, K., Pelcat, M., Nezan, J., Aridhi, S.: Memory bounds for the distributed execution of a hierarchical synchronous data-flow graph. In: Embedded Computer Systems (SAMOS), 2012 International Conference on (2012)

7. Desnos, K., Pelcat, M., Nezan, J.F., Aridhi, S.: Pre-and post-scheduling memory allocation strategies on mpsocs. In: Electronic System Level Synthesis Conference (ESLsyn) (2013)

8. Desnos, K., Zhang, J.: Preesm project - stereo matching (2013). URL svn://svn.code.sf.net/p/preesm/code/trunk/tests/stereo

9. El Assad, S., Noura, H.: Generator of chaotic sequences and corresponding generating system (2013). URL http://www.google.com/patents/EP2553567A1?cl=en. EP Patent App. EP20,110,720,313

10. Electronic Systems Group TU Eindhoven, .: Sdf for free (sdf3) (2013). URL http://www.es.ele.tue.nl/sdf3/

11. Embedded Vision Alliance, .: Embedded vision alliance (2013). URL http://www.embedded-vision.com

12. Fabri, J.: Automatic storage optimization. Courant Institute of Mathematical Sciences, New York University (1979)

13. Fischaber, S., Woods, R., McAllister, J.: Soc memory hierarchy derivation from dataflow graphs. In: Signal Processing Systems, 2007 IEEE Workshop on, pp. 469-474 (2007). DOI 10.1109/SIPS.2007.4387593

14. Greef, E.D., Catthoor, F., Man, H.D.: Array placement for storage size reduction in embedded multimedia systems. ASAP (1997)
15. Intel: i7-3610qm processor product page (2013). URL http://ark.intel.com/products/64899/

16. Johnson, D.S.: Near-optimal bin packing algorithms. Ph.D. thesis, Massachusetts Institute of Technology (1973)

17. Kalray: Many-core processors - dataflow (2013). URL http://www.kalray.eu/technology/dataflow/

18. Lee, E., Messerschmitt, D.: Synchronous data flow. Proceedings of the IEEE 75(9), 1235 - 1245 (1987). DOI 10.1109/PROC.1987.13876

19. Lee, E.A., Parks, T.M.: Dataflow process networks. Proceedings of the IEEE 83(5), 773-801 (1995)

20. Malamas, E.N., Petrakis, E.G., Zervakis, M., Petit, L., Legat, J.D.: A survey on industrial vision systems, applications and tools. Image and vision computing 21(2), 171-188 (2003)

21. Murthy, P., Bhattacharyya, S.: Shared memory implementations of synchronous dataflow specifications. In: Design, Automation and Test in Europe Conference and Exhibition 2000. Proceedings (2000)

22. Murthy, P.K., Bhattacharyya, S.S.: Memory management for synthesis of DSP software. CRC Press (2010)

23. Östergård, P.R.J.: A new algorithm for the maximum weight clique problem. Nordic J. of Computing (2001)

24. Parks, T.M.: Bounded scheduling of process networks. Ph.D. thesis, University of California (1995)

25. Pelcat, M., Aridhi, S., Piat, J., Nezan, J.F.: Physical Layer Multi-Core Prototyping: A Dataflow-Based Approach for LTE eNodeB. Springer (2012)

26. Pelcat, M., Nezan, J.F., Piat, J., Croizer, J., Aridhi, S.: A System-Level architecture model for rapid prototyping of heterogeneous multicore embedded systems. DASIP (2009)

27. Roy, S.: Stereo without epipolar lines: A maximum-flow formulation. International Journal of Computer Vision 34(2-3), 147-161 (1999)

28. Sriram, S., Bhattacharyya, S.S.: Embedded Multiprocessors: Scheduling and Synchronization, 2nd edn. CRC Press, Inc., Boca Raton, FL, USA (2009)

29. Stuijk, S., Geilen, M., Basten, T.: Exploring trade-offs in buffer requirements and throughput constraints for synchronous dataflow graphs. In: Proceedings of the 43rd annual Design Automation Conference (2006)

30. Szeliski, R., Zabih, R.: An experimental comparison of stereo algorithms. In: Vision Algorithms: Theory and Practice, pp. 1-19. Springer (2000)

31. Szymanek, R., Kuchcinski, K.: A constructive algorithm for memory-aware task assignment and scheduling. In CODES Proceedings (2001)

32. Texas Instruments, .: Tms320c6678 product page (2013). URL http://www.ti.com/product/tms320c6678

33. Urban, F., Raulet, M., Nezan, J.F., Déforges, O.: Automatic dsp cache memory management and fast prototyping for multiprocessor image applications. In: 14th European Signal Processing Conference, Eusipco (2006)

34. Wagner, D.: Handheld augmented reality. Ph.D. thesis, Graz University of Technology (2007)

35. Wulf, W.A., McKee, S.A.: Hitting the memory wall: implications of the obvious. ACM SIGARCH computer architecture news 23(1), 20-24 (1995)

36. Yamaguchi, K., Masuda, S.: A new exact algorithm for the maximum weight clique problem. In: 23rd International Conference on Circuit/Systems, Computers and Communications (ITC-CSCC'08) (2008)

37. Zhang, J., Nezan, J.F., Pelcat, M., Cousin, J.G.: Realtime gpu-based local stereo matching method. In: Design and Architectures for Signal and Image Processing (DASIP), 2013 Conference on, pp. 209-214. IEEE (2013) 